\title{
Design and manufacturing process for the KIT 2-MW 170-GHz coaxial-cavity longer-pulse gyrotron
}

\author{
$\underline{\text { S. Ruess }}{ }^{1,2}$, G. Gantenbein ${ }^{1}$, A. Heinzel ${ }^{1}$, S. Illy ${ }^{1}$, I. Gr. Pagonakis ${ }^{1}$, T. Rzesnicki ${ }^{1}$, \\ M. Thumm ${ }^{1,2}$, J. Weggen ${ }^{1}$, A. Weisenburger ${ }^{1}$, and J. Jelonnek ${ }^{1,2}$ \\ ${ }^{1} \mathrm{IHM},{ }^{2} \mathrm{IHE}$, Karlsruhe Institute of Technology (KIT), 76131 Karlsruhe, Germany \\ Sebastian.Ruess@kit.edu
}

Today's performance requirements for future DEMO gyrotrons are an operating frequency between $170 \mathrm{GHz}$ up to $240 \mathrm{GHz}$ and an output power of significantly larger than $1 \mathrm{MW}$ (today's target: $2 \mathrm{MW}$ ). A total gyrotron efficiency of better than $60 \%$ must be achieved. Multipurpose/multi-frequency operation and frequency steptunability are required also. It has been shown earlier, that the coaxial-cavity technology is a promising candidate [1]. In [2] a world record RF output power of $2.2 \mathrm{MW}$ has been reported for short-pulses (in the range of few milliseconds). Nevertheless, it has to be proven that the coaxial-cavity technology can be used for long-pulse operation. That shall be achieved by upgrading the existing $2 \mathrm{MW} 170 \mathrm{GHz}$ short-pulse prototype used for the demonstration of the $2.2 \mathrm{MW}$ record power. Additionally, a new Inverse Magnetron Injection Gun (IMIG) shall allow a significant larger emitter radius and therefore increased output power at operating frequencies significantly above $200 \mathrm{GHz}$ by keeping the same or even smaller diameter of the warm bore hole of the superconducting gyrotron magnet. Both, the IMIG as well as the longer pulse gyrotron will show the way towards higher output power at higher operating frequencies of gyrotrons together with a more robust design and construction of that kind of tubes.

\section{Heading towards a longer-pulse tube}

Fig. 1 shows the major upgraded parts of the existing KIT modular coaxial-cavity tube $[3,4]$. In detail, for the longer-pulse tube a new modular system concept for cooling the beam tunnel, the cavity and the launcher has been developed. Fully independent water cycles for each of the named gyrotron components have been designed and verified with the multi-physics software Comsol. This concept allows the monitoring of the internal losses in each gyrotron component and of the final energy balance of the tube during long pulse operation. It has to be mentioned, that the longer-pulse $2 \mathrm{MW}$ gyrotron is currently under manufacturing in the KIT workshop and the first operation is planned for mid-2017.

\section{Introduction to the advanced IMIG}

The presented Inverse Magnetron Injection gun as well as the longer pulse gyrotron will be a major step towards higher output power. Due to the larger effective emitter radius the IMIG can be operated at higher output power levels at operating frequencies above $200 \mathrm{GHz}$ by keeping the same or even smaller diameter of the warm bore hole of the superconducting magnet. At these very high frequencies, magnetic field strengths of above $10 \mathrm{~T}$ have to be considered for the magnet. Furthermore, the advanced IMIG design is of modular type. It allows the implementation and test of new emitter technologies with significantly increased current density. The proposed design of the IMIG [1] matches the design and the nominal operating parameters of the European 170-GHz, 2MW coaxial-cavity gyrotron, originally intended for the first installation at ITER [2]. The IMIG can also be operated in the KIT 2-MW coaxial longer-pulse gyrotron [5], as well as in conventional gyrotrons [6] and is compatible with future fusion gyrotrons at $204 \mathrm{GHz}$ and multi-MW output power range.

\section{Realization of vacuum-tight connections}

One of the most critical issues in a gyrotron are the high-vacuum compatible brazed and soldered joints. OM (optical microscopy) (see Fig. 3) and SEM (Scanning Electron Microscope) cross sections and measurements of different brazed and soldered joints with variable material compositions have shown excellent vacuum compatible connections with a He leakage rate of $<10-10 \mathrm{mbar} 1 / \mathrm{s}$. In Fig. 3, cross sections of different brazed and soldered joints with variable material compositions are shown. The two at the top in Fig. 3 show a soldered joint with Glidcop and stainless steel. It can be very well seen that the solder flowed into the gap due to the capillary forces and diffuses into the Glidcop and stainless steel. In addition, excellent solder joints between CVD diamond and copper were also achieved.

\section{Experimental Measurements}

One of the main advantages of the IMIG is the optimized cooling concept of the cathode, especially for high thermally loaded components in the neighboring emitter regions and at the anode. Therefore, the thermal expansion in the IMIG could be significantly reduced.

The corresponding simulation results were already presented in [5]. In order to verify the thermomechanical simulation results a measurement setup was designed and manufactured. By using an IR-camera the temperature distribution at the neighboring region of the emitter will be measured and compared with the simulation results to verify the assumptions for the thermomechanical simulations. In the case of the IMIG, the cathode can be used as a vacuum chamber, which is closed on the top by a $\mathrm{ZnS}$ $\mathrm{CG}$ window. The measurement system is closed with a blind flange and intake socket for continuous vacuum pumping at its bottom. At the same time, the measurement system contributes also to the calibration of the filament power versus the emitter temperature.

It shall be pointed out that the IMIG is the first multiMW Magnetron Injection Gun, which is in-house manufactured at KIT. The emitter ring is produced by $3 \mathrm{M}$, Ceradyne Inc. Measurements of the already produced com- 
ponents indicate excellent surface conditions and small tolerances $(<20 \mu \mathrm{m})$ which permits a homogenous electric field at the emitter region and therefore a high quality electron beam.

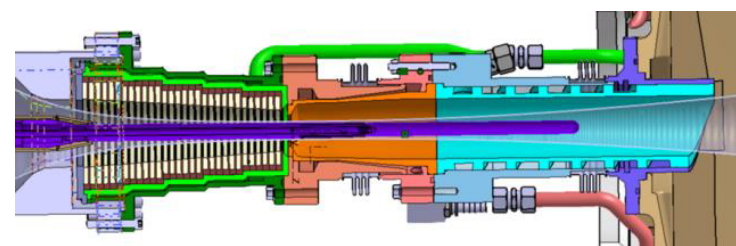

Fig. 1. Sketch of the KIT modular longer-pulse (expected pulse lengths $100 \mathrm{~ms}-1 \mathrm{~s}$ ) gyrotron pre-prototype. Major parts are the beam tunnel (green), the cavity (orange) and the launcher (blue)

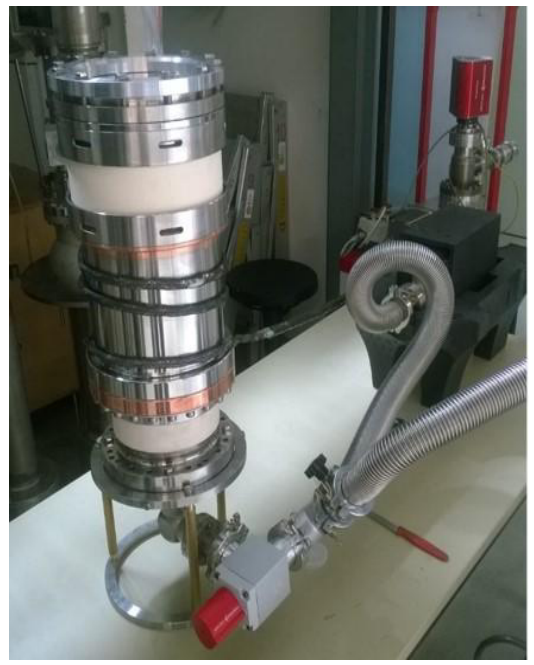

Fig. 2. Assembled Inverse Magnetron Injection Gun during the bake out process
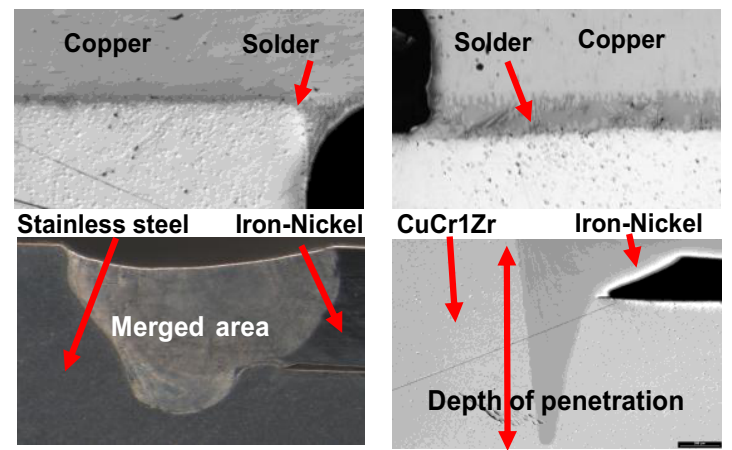

Fig. 3. Cross sections of brazed and soldered joints (OM and SEM)

\section{Summary and Outlook}

The already successfully manufactured parts of the KIT longer-pulse coaxial-cavity gyrotron are presented. In particular the construction of an innovative IMIG is discussed. The IMIG is prepared for the use with the KIT $2 \mathrm{MW} / 170 \mathrm{GHz}$ coaxial-cavity gyrotron. The IMIG permits an optimized cooling concept of thermally loaded components and allows for a given warm bore hole radius of the gyrotron magnet a significantly larger emitter ring compared to "conventional" MIGs. In addition, the IMIG is completely in-house manufactured with excellent surface quality and small tolerances. Due to the optimized construction of the beam-forming components, the IMIG was easily re-machined after the implementation of the ceramics, which results in smaller tolerances. To verify the thermomechanical simulation results, a comparison of the temperature distribution of the IMIG between the experimental results and the simulation results will be made by the use of a measurement test facility in mid2017.

\section{Acknowledgements}

Part of this work is carried out within the framework of the EUROfusion Consortium and is receiving funding from the Euratom research and training programme 20142018 under grant agreement No 633053. The views and opinions expressed herein do not necessarily reflect those of the European Commission.

\section{References}

1. B. Piosczyk, et al. "A 2-MW, 170-GHz Coaxial Cavity Gyrotron". IEEE Transaction on Electron Devices, (32):413417, June 2004.

2. T. Rzesnicki, et al. IEEE Trans. Plasma Science, Vol. 38, No. 6, pp. 1141-1149, 2010.

3. S. Ruess, et al. "Experimental results and outlook of the $2 \mathrm{MW} 170 \mathrm{GHz}$ coaxial-cavity gyrotron towards long pulse operation", German Microwave Conference (GeMiC) 2016, Bochum, Germany, pp. 369-372,2016.

4. S. Ruess et al. „Coaxial Gyrotron Development: From ITER towards DEMO “, Proc. European Microwave Conference (EuMC ) 2017, Nuernberg, Germany, 2017.

5. S. Ruess, et al. „An Inverse Magnetron Injection Gun for the KIT 2-MW Coaxial-Cavity Gyrotron", IEEE Transactions on Electron Devices, (63), p. 2104-2109, 2016, DOI:10.1109/ TED.2016.2540298.

6. S.Ruess, et al. Proposal of an Inverse Magnetron Injection Gun for Future Hollow-Cylindrical-Cavity High Power Gyrotrons" Proc. IRMMW 2016, Copenhagen, Denmark, September 2016, W4C. 